Journal of Bangladesh Academy of Sciences, Vol. 35, No. 1, 43-50, 2011

\title{
APPLICATION OF SCALING GROUP OF TRANSFORMATIONS TO STEADY BOUNDARY LAYER FLOW OF NEWTONIAN FLUID OVER A STRETCHING SHEET IN PRESENCE OF CHEMICALLY REACTIVE SPECIES
}

\author{
K. BHATTACHARYYA, M. S. UDDIN ${ }^{* 1}$ AND G. C. LAYEK \\ Department of Mathematics, The University of Burdwan, Burdwan-713104, West Bengal, \\ India
}

\begin{abstract}
This investigation analyses application of Lie's scaling group of transformations to steady flow of a Newtonian fluid over a stretching sheet in presence of chemically reactive species with first order reaction. The governing partial differential equations reduced to self-similar nonlinear ordinary differential equations by the transformations. Obtained momentum equation is solved analytically and the concentration equation is numerically solved applying finite difference method with Thomas algorithm. The plotted results reveal that with the increase of Schmidt number as well as reaction-rate parameter causes a reduction in the thickness of the concentration boundary layer and also the concentration at a point decreases.
\end{abstract}

Key words: Scaling group of transformation, Boundary layer flow, Newtonian fluid, Stretching sheet, Chemically reactive species

\section{INTRODUCTION}

The flow due to linearly stretching sheet is a standard problem in fluid mechanics. The problem has huge practical applications in polymer processing industries, paper production, several biological process and many others. The diffusion of chemically reactive species in boundary layer flow around the stretching sheet is very important in chemical industries, metal and polymer processing industries.

Crane (1970) was the first person to study the laminar boundary layer flow caused by a stretching sheet with stretching velocity varying linearly with distance from a fixed point. The heat and mass transfer in Newtonian boundary layer flow past a stretching sheet with suction or blowing was studied by Gupta and Gupta (1977). Chakrabarti and Gupta (1979) analyzed the magnetohydrodynamics (MHD) flow of Newtonian fluid initially at rest over a stretching sheet at different values of the parameter related with the uniform temperature. Grubka and Bobba (1985) investigated the heat transfer characteristics of the stretching sheet problem with variable temperature. Tapanidis et al. (2003) discussed the application of scaling group of transformations to visco-elastic second-grade fluid flow. Recently, Mukhopadhyay et al. (2005) studied the MHD boundary layer flow and heat transfer over a stretching sheet with variable viscosity using the scaling group of transformations.

* Corresponding author: <sharif_ku@yahoo.com.>.

${ }^{1}$ Mathematics Discipline, Khulna University, Khulna-9208, Bangladesh. 
However, there are a several investigations on chemical reaction effects on flowing fluid done by many researchers. Das et al. (1994) investigated the effect of mass transfer on flow past an impulsively started infinite vertical plate with constant heat flux and chemical reaction. Anderson et al. (1994) studied the diffusion of a chemically reactive species from a stretching sheet. The similarity solutions of mixed convection with diffusion and chemical reaction over a horizontal moving plate were obtained by Fan $e t$ al. (1998). The study of MHD free convective flow and mass transfer over a stretching sheet with chemical reaction was done by Afify (2004). Recently, Cortell (2007a,b) discussed the effects of magnetic field on the flow and mass transfer of second grade fluid in a porous medium over a stretching sheet with chemically reactive species and also analyzed the motion and mass transfer for two classes of viscoelastic fluid past a porous stretching sheet with suction or blowing.

In this paper, authors shall concentrate on the application of scaling group of transformations to analyze the steady boundary layer flow past a stretching sheet in presence of chemically reactive species, under going a first order reaction. By applying suitable scaling group of transformations, the set of governing partial differential equations for the flow and concentration distribution are transformed into a set of selfsimilar ordinary differential equations. The appropriate boundary conditions for the velocity and mass concentration fields are also transformed. The solution of the momentum equation is obtained analytically and the concentration equation is solved numerically by finite difference method using Thomas algorithm. The analytical as well as numerical solutions have been plotted for different values of parameters involved.

\section{FORMATION OF THE PROBLEM}

Authors consider the steady flow of a viscous incompressible Newtonian fluid past a stretching sheet in presence of chemically reactive species under going a first order reaction. Using boundary layer approximation, the governing continuity, momentum and concentration equations in the may be written in usual notation as :

$$
\begin{gathered}
\frac{\partial u}{\partial x}+\frac{\partial v}{\partial y}=0 \\
u \frac{\partial u}{\partial x}+v \frac{\partial v}{\partial y}=v \frac{\partial^{2} v}{\partial y^{2}}, \\
\text { and } u \frac{\partial C}{\partial x}+v \frac{\partial C}{\partial y}=D \frac{\partial^{2} C}{\partial y^{2}}-k C
\end{gathered}
$$

where $u$ and $v$ are the components of the fluid velocity along the $x$ and $y$ directions respectively, $v$ is the kinematic viscosity, $C$ is the concentration, $D$ is the diffusion coefficient and $k$ denotes the reaction rate constant. 
The corresponding boundary conditions are given as :

$$
\begin{aligned}
& u=a x \text { and } v=0 \text { at } y=0 \text { and } u \rightarrow 0 \text { as } y \rightarrow \infty ; \\
& C=C_{w} \text { at } y=0 \text { and } C \rightarrow 0 \text { as } y \rightarrow \infty,
\end{aligned}
$$

where $a>0$ is stretching constant, $C_{w}$ denotes the concentration at the stretching sheet.

Authors now introduce the stream function $\psi$ as :

$$
u=\frac{\partial \psi}{\partial y} \text { and } v=\frac{\partial \psi}{\partial x}
$$

and a relation for the concentration as :

$$
C=C_{w} \bar{C} .
$$

The continuity Eq. (1) is satisfied clearly by the relations (6). In view of the relations (6) and (7), the Eqs. (2) and (3) reduce respectively to

$$
\begin{aligned}
& \frac{\partial \psi}{\partial y} \frac{\partial^{2} \psi}{\partial x \partial y}-\frac{\partial \psi}{\partial x} \frac{\partial^{2} \psi}{\partial y^{2}}=v \frac{\partial^{3} \psi}{\partial y^{3}} \\
& \frac{\partial \psi}{\partial y} \frac{\partial \bar{C}}{\partial x}-\frac{\partial \psi}{\partial x} \frac{\partial \bar{C}}{\partial y}=D \frac{\partial^{2} \bar{C}}{\partial y^{2}}-k \bar{C}
\end{aligned}
$$

and the boundary conditions become

$$
\begin{aligned}
& \frac{\partial \psi}{\partial y}=a x, \frac{\partial \psi}{\partial x}=0 \text { at } y=0 \text { and } \frac{\partial \psi}{\partial y} \rightarrow 0 \text { at } y \rightarrow \infty ; \\
& \bar{C}=1 \text { at } y=0 \text { and } \bar{C} \rightarrow 0 \text { at } y \rightarrow \infty .
\end{aligned}
$$

\section{SCALING GROUP OF TRANSFORMATIONS}

Authors now introduce the simplified form of Lie-group transformations, namely, the scaling group of transformations (Tapanidis et al. 2003, Mukhopadhyay et al. 2005) as :

$$
\Gamma: x^{*}=x e^{\varepsilon \alpha_{1}}, y^{*}=y e^{\varepsilon \alpha_{2}}, \psi^{*}=\psi e^{\varepsilon \alpha_{3}}, u^{*}=u e^{\varepsilon \alpha_{4}}, v^{*}=v e^{\varepsilon \alpha_{5}} \text { and } C^{*}=\bar{C} e^{\varepsilon \alpha_{6}}
$$

The transformation (12) may be considered as a point transformation, which transformed the coordinates $(x, y, \psi, u, v, \bar{C})$ to the coordinates $\left(x^{*}, y^{*}, \psi^{*}, u^{*}, v^{*}, C^{*}\right)$.

Taking the relations (12) in to account in Eqs. (12) and (13), authors obtain, respectively

$$
e^{\varepsilon\left(\alpha_{1}+2 \alpha_{2}-2 \alpha_{3}\right)}\left(\frac{\partial \psi^{*}}{\partial y^{*}} \frac{\partial^{2} \psi^{*}}{\partial x^{*} \partial y^{*}}-\frac{\partial \psi^{*}}{\partial x^{*}} \frac{\partial^{2} \psi^{*}}{\partial y^{* 2}}\right)=e^{\varepsilon\left(3 \alpha_{2}-\alpha_{3}\right)} v \frac{\partial^{3} \psi^{*}}{\partial y^{* 3}}
$$


and $e^{\varepsilon\left(\alpha_{2}-\alpha_{3}-\alpha_{6}\right)} x^{*}\left(\frac{\partial \psi^{*}}{\partial y^{*}} \frac{\partial C^{*}}{\partial x^{*}}-\frac{\partial \psi^{*}}{\partial x^{*}} \frac{\partial C^{*}}{\partial y^{*}}\right)=e^{\varepsilon\left(2 \alpha_{2}-\alpha_{1}-\alpha_{6}\right)} x^{*} D \frac{\partial^{2} C^{*}}{\partial y^{* 2}}-e^{-\varepsilon\left(\alpha_{1}+\alpha_{6}\right)} k x^{*} C^{*}$

Both equations will remain invariant under the scaling group of transformations $\Gamma$, if the following relations among the transformation parameters hold.

$$
\left.\begin{array}{c}
\alpha_{1}+2 \alpha_{2}-2 \alpha_{3}=3 \alpha_{2}-\alpha_{3} \\
\text { and } \alpha_{2}-\alpha_{3}-\alpha_{6}=2 \alpha_{2}-\alpha_{1}-\alpha_{6}=-\alpha_{1}-\alpha_{6}
\end{array}\right\}
$$

From (15) authors can easily obtain $\alpha_{2}=0$ and $\alpha_{1}=\alpha_{3}$. The relation $u^{*}=\partial \psi^{*} / \partial y^{*}$ and $v^{*}=-\partial \psi^{*} / \partial x^{*}$ gives us $\alpha_{3}=\alpha_{4}, \alpha_{5}=0$. In view of these, the boundary conditions (10) and (11) are transformed to

$$
\begin{aligned}
& \frac{\partial \psi^{*}}{\partial y^{*}}=a x^{*}, \frac{\partial \psi^{*}}{\partial x^{*}}=0 \text { at } y^{*}=0 \text { and } \frac{\partial \psi^{*}}{\partial y^{*}} \rightarrow 0 \text { as } y^{*} \rightarrow \infty ; \\
& C^{*}=1 \text { at } y^{*}=0 \text { and } C^{*} \rightarrow 0 \text { as } y^{*} \rightarrow \infty,
\end{aligned}
$$

where the boundary condition $C^{*}=1$ gives $\alpha_{6}=0$.

Thus the set of transformations $\Gamma$ finally reduces to a one-parameter group of transformations

$$
\Gamma: x^{*}=x e^{\varepsilon \alpha_{1}}, y^{*}=y, \psi^{*}=\psi e^{\varepsilon \alpha_{1}}, u^{*}=u e^{\varepsilon \alpha_{1}}, v^{*}=v \text { and } C^{*}=\bar{C}
$$

Firstly, authors consider the absolute invariant, $\eta$ which is a function of the independent variables and is taken as $\eta=y^{*} x^{* p}$.

Since the quantity $\eta$ is absolute invariant, they get $y^{*} x^{* p}=y x^{p}$.

Now, $y^{*} x^{* p}=y x^{p} e^{\varepsilon \alpha_{1} p}=y x^{p}$ if $p=0\left(\right.$ since $\alpha_{1}$ cannot be 0$)$

Hence, authors ultimately get the first absolute invariant as $\eta=y^{*}$.

Authors now calculate the second absolute invariant, $G=f(\eta)$ which involves the dependent variable $\psi^{*}$ and assume that $G=x^{* q} \psi^{*}$. Since $G$ is an absolute invariant, we will find $q$ such that $x^{* q} \psi^{*}=x^{q} \psi$.

Now, $x^{* q} \psi^{*}=\left(x e^{\varepsilon \alpha_{1}}\right)^{q} \psi e^{\varepsilon \alpha_{1}}=\left(x^{q} e^{\varepsilon \alpha_{1} q}\right) \psi e^{\varepsilon \alpha_{1}}=e^{\varepsilon \alpha_{1}(q+1)} x^{q} \psi=x^{q} \psi$ if $q=-1$.

Putting $q=-1$, the second absolute invariant $G$ becomes

$G=x^{*-1} \psi^{*}$ i.e. $f(\eta)=x^{*-1} \psi^{*}$.

Lastly, authors attention is concentrated in finding the third absolute invariant, $H=\phi$ $(\eta)$ which involves the independent variables and the dependent variable $C^{*}$ and is taken as $H=x^{* r} C^{*} . H$ is an absolute invariant if $x^{* r} C^{*}=x^{r} \bar{C}$. 
Now, $x^{{ }^{*} r} C^{*}=\left(x e^{\varepsilon \alpha_{1}}\right)^{r} \bar{C}=\left(x^{r} e^{\varepsilon \alpha_{1} r}\right) \bar{C}=e^{\varepsilon \alpha_{1} r} x^{r} \bar{C}=x^{r} \bar{C}$ if $r=0$.

Thus, the third absolute invariant is $H=C^{*}$ i.e. $\phi(\eta)=C^{*}$.

Finally, from three absolute invariants, they get the following transformations as:

$\eta=y^{*}, \psi^{*}=x^{*} f(\eta)$ and $C^{*}=\phi(\eta)$.

In view of the above relations, the Eqs. (13) and (14) become

$v f^{\prime \prime \prime}+f f^{\prime \prime}-f^{\prime 2}=0$

and $D \phi^{\prime \prime}+f \phi^{\prime}-k \phi=0$

and the corresponding boundary conditions (16) and (17) are reduced to

$$
\begin{aligned}
& f(\eta)=0, f^{\prime}(\eta)=a \text { at } \eta=0 \text { and } f^{\prime}(\eta) \rightarrow 0 \text { as } \eta \rightarrow \infty ; \\
& \phi(\eta)=1 \text { at } \eta=0 \text { and } \phi(\eta) \rightarrow 0 \text { as } \eta \rightarrow \infty
\end{aligned}
$$

Again, to eliminate the constant $a$ and $v$ authors introduce the following transformations for $\eta, f$ and $\phi$ in Eqs. (20) - (23):

$$
\eta=v^{\alpha^{\prime}} a^{\beta^{\prime}} \bar{\eta}, f=v^{\alpha^{\prime \prime}} a^{\beta^{\prime \prime}} \bar{f} \text { and } \phi=v^{\alpha^{\prime \prime \prime}} a^{\beta^{\prime \prime \prime}} \bar{\phi}
$$

and they obtain $\alpha^{\prime}=\alpha^{\prime \prime}=1 / 2, \beta^{\prime}=1 / 2, \beta^{\prime \prime}=-1 / 2$ and $\alpha^{\prime \prime \prime}=\beta^{\prime \prime \prime}=0$.

Finally, in view of the above transformations and taking $\bar{\eta}=\eta, \bar{f}=f$ and $\bar{\phi}=\phi$ the Eqs. (24) and (25) are reduced to the following self-similar forms:

$f^{\prime \prime \prime}+f f^{\prime \prime}-f^{\prime 2}=0$

and $\phi^{\prime \prime}+S c f \phi^{\prime}-S c \beta \phi=0$,

where $S c=v / D$ is the Schmidt number and $\beta=k / a$ is reaction rate parameter of the solute.

The appropriate boundary conditions (22) and (23) take the following forms:

$$
\begin{aligned}
& f(\eta)=0, f^{\prime}(\eta)=1 \text { at } \eta=0 \text { and } f^{\prime}(\eta) \rightarrow 0 \text { as } \eta \rightarrow \infty ; \\
& \phi(\eta)=1 \text { at } \eta=0 \text { and } \phi(\eta) \rightarrow 0 \text { as } \eta \rightarrow \infty \text {. }
\end{aligned}
$$

\section{SOLUTION OF PROBLEM}

Crane (1970) obtained the exact analytical solution of equation (25) satisfying boundary conditions (27) as

$$
f(\eta)=1-\exp (-\eta) \text {. }
$$

Now, the equation (26) transfers to the form given by

$$
\phi^{\prime \prime}+S c\left(1-e^{-\eta}\right) \phi^{\prime}-S c \beta \phi=0
$$


satisfying the boundary conditions (28).

Authors have to choose a suitable finite value of $\eta \rightarrow \infty$, say $\eta^{*}$ and then the interval $\left[0, \eta^{*}\right]$ is divided into $N$ equal subintervals of length $\Delta \eta=0.01$ taking $\eta^{*}=20$ for all the cases. Applying central finite difference formulae for the second and first orders derivatives of $\phi$ as

$$
\phi^{\prime \prime}=\frac{\phi_{j+1}-2 \phi_{j}+\phi_{j-1}}{(\Delta \eta)^{2}} \text { and } \phi^{\prime}=\frac{\phi_{j+1}-\phi_{j-1}}{2(\Delta \eta)}
$$

the above equation (30) along with the boundary condition (28) reduce to

$$
\begin{aligned}
& \phi_{j-1} a_{j}+\phi_{j} b_{j}+\phi_{j+1} c_{j}=0,1 \leq j \leq N \\
& \text { with } \phi_{0}=1 \text { and } \phi_{N+1}=0
\end{aligned}
$$

where $a_{j}=\frac{1}{(\Delta \eta)^{2}}-\frac{S c\left(1-e^{-\eta_{j}}\right)}{2(\Delta \eta)}, b_{j}=-\frac{2}{(\Delta \eta)^{2}}-S c \beta, c_{j}=\frac{1}{(\Delta \eta)^{2}}-\frac{S c\left(1-e^{-\eta_{j}}\right)}{2(\Delta \eta)}$;

$$
1 \leq j \leq N
$$

Authors solve the system of equations (31) with the conditions (32) by the standard Thomas algorithm for solving the tri-diagonal system.

\section{RESULTS AND DISCUSSION}

The analytic solution of velocity field and numerical solution of concentration field for various values of parameters viz. Schmidt number and reaction-rate parameter are plotted in figures and discussed physically in every aspect.

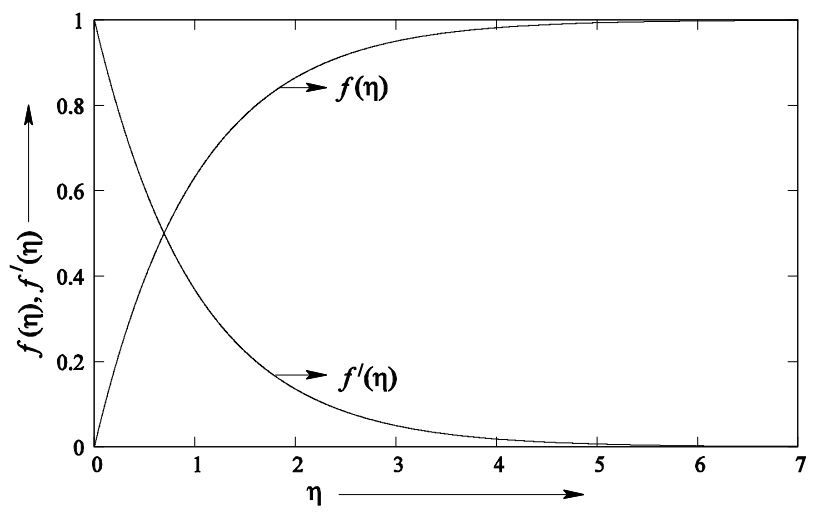

Fig. 1. The dimensionless velocity $f^{\prime}(\eta)$ and stream function $f(\eta)$.

The analytic solution for dimensionless velocity $f^{\prime}(\eta)$ and stream function $f(\eta)$ are plotted in Fig. 1 which shows the behaviour of flow inside the boundary layer. For large value of $\eta$ the velocity diminishes, which is compatible with physical nature of the flow. 
Authors now focus their attention on the variation of concentration profiles for several values of Schmidt number Sc. Fig. 2 represents the concentration profiles for different values of Schmidt number. From the figure it is seen that the concentration at a point as well as the thickness of concentration boundary layer decrease due to the increase of $S c$. The Schmidt number is inversely proportional to the diffusion coefficient.

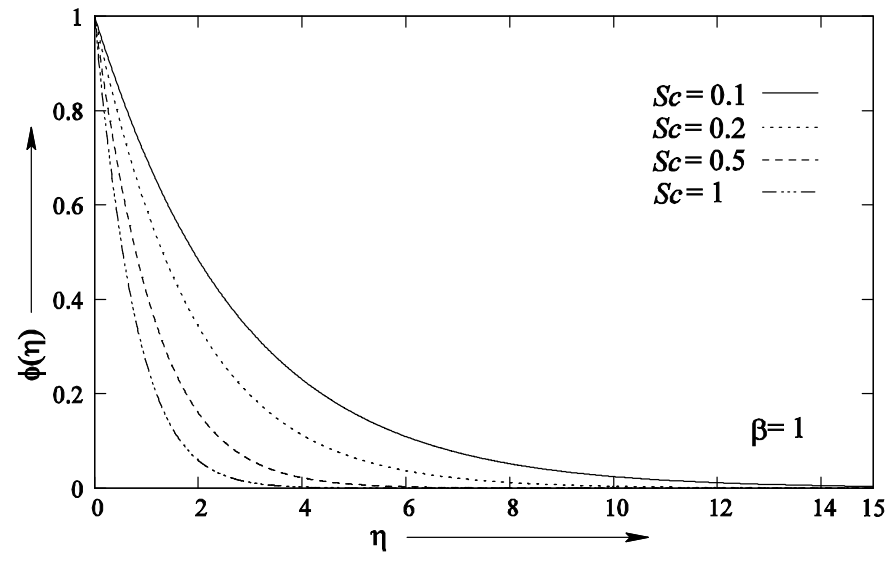

Fig. 2. Concentration profiles $\phi(\eta)$ for various values of $S c$ with $\beta=1$.

So, increase of diffusion coefficient increases the solute boundary layer thickness. At large values $\eta$, the solute distribution vanish as expected. Finally, authors now discuss the effect of reaction-rate parameter $\beta$ on the reactive concentration profiles. Fig. 3 is the graphical picture of concentration profiles for various values of $\beta$.

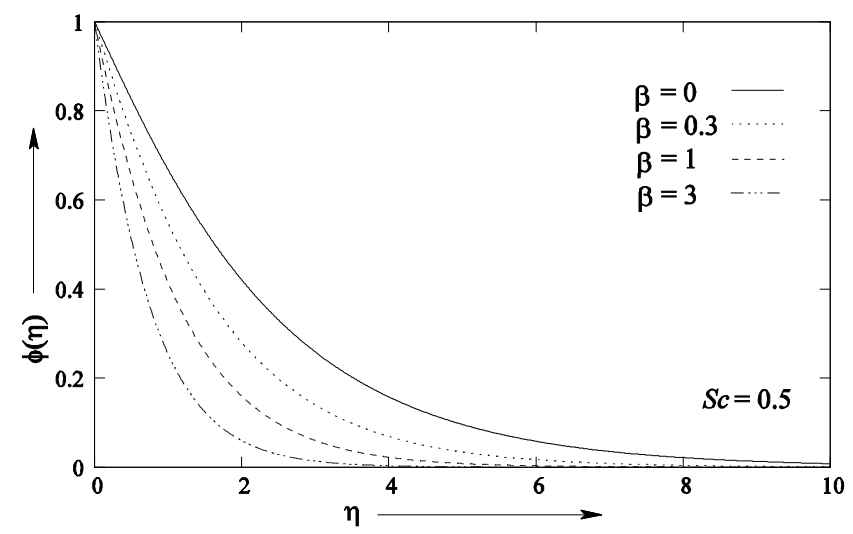

Fig. 3. Concentration profiles $\phi(\eta)$ for various values of $\beta$ with $S c=0.5$.

Various curves representing the concentration profiles $\phi(\eta)$ for several values of $\beta$ demonstrate that the value of reactive concentration at a fixed $\eta$ decreases with increasing $\beta$. So, the reaction-rate parameter is a decelerating agent and it thins the solute boundary layer formed near the stretching wall. 


\section{CONCLUSIONS}

The application of scaling group of transformations to steady boundary layer flow of viscous incompressible fluid over a stretching sheet in presence of chemically reactive species is demonstrated. After the transformations, the reduced self-similar ordinary differential equations are solved. The exact solution for velocity field is obtained and for the concentration distribution a numerical solution is found. The study shows that the concentration boundary layer thickness decreases with the increase of Schmidt number as well as reaction-rate parameter.

\section{ACKNOWLEDGEMENTS}

The authors express their gratitude to the reviewer for his valuable comments and suggestions. One of the authors (KB) gratefully acknowledges the financial support of National Board for Higher Mathematics (NBHM), DAE, Mumbai, India for pursuing this work.

\section{REFERENCES}

Afify, A. 2004. MHD free convective flow and mass transfer over a stretching sheet with chemical reaction. Heat Mass Trans. 40:495-500.

Anderson, H. I., O. R. Hansen and B. Holmedal. 1994. Diffusion of a chemically reactive species from a stretching sheet. Int. J. Heat Mass Trans. 37:659-664.

Chakrabarti, A. and A.S. Gupta. 1979. Hydromagnetic flow and heat transfer over a stretching sheet. Q. Appl. Math. 37:73-78.

Cortell, R. 2007a. MHD flow and mass transfer of an electrically conducting fluid of second grade in a porous medium over a stretching sheet with chemically reactive species. Chem. Eng. Process. 46:721-728.

Cortell, R. 2007b. Toward an understanding of the motion and mass transfer with chemically reactive species for two classes of viscoelastic fluid over a porous stretching sheet. Chem. Eng. Process. 46:982-989.

Crane, L. J. 1970. Flow past a stretching plate. ZAMP 21:645-647.

Das, U. N., R. Deka and V. M. Soundalgekar. 1994. Effect of mass transfer on flow past an impulsively started infinite vertical plate with constant heat flux and chemical reaction. Forsch. Ingenieurwes. 60:284-287.

Fan, J. R., J. M. Shi and X. Z. Xu. 1998. Similarity solution of mixed convection with diffusion and chemical reaction over a horizontal moving plate. Acta Mech. 126:59-69.

Grubka, L. J. and K. M. Bobba. 1985. Heat transfer characteristics of a continuous, stretching surface with variable temperature. ASME J. Heat Trans. 107:248-250.

Gupta, P. S. and A. S. Gupta. 1977. Heat and mass transfer on a stretching sheet with suction and blowing. Can. J. Chem. Eng. 55:744-746.

Mukhopadhyay, S., G. C. Layek and Sk. A. Samad. 2005. Study of MHD boundary layer flow over a heated stretching sheet with variable viscosity. Int. J. Heat Mass Trans. 48:4460-4466.

Tapanidis, T., G. Tsagas and H. P. Mazumdar. 2003. Application of scaling group of transformations to visco-elastic second-grade fluid flow. Nonlinear Funct. Anal. Appl. 8:345-350. 